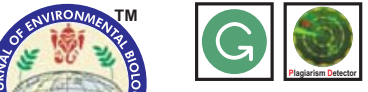

\title{
Identification of dehydrin markers in Capsicum annuum for development of drought tolerant crops in changing environment
}

Authors Info

R. R. Thomas', M. K. C. Prakash', M. K. Reddy', R. Mahmood ${ }^{3}$ and P. Mondal'

${ }^{1}$ Division of Social Sciences, Indian Institute of Horticultural Research, Bengaluru-560 089, India

${ }^{2}$ Division of Plant Pathology, Indian Institute of Horticultural Research, Bengaluru-560 089, India

${ }^{3}$ Department of Biotechnology and Bioinformatics, Kuvempu University, Shivamogga-577451, India

*Corresponding Author Email : reenart@hotmail.com

Key words

Capsicum annuum,

Dehydrin marker,

Drought tolerance,

EST sequences,

Plant breeding

Publication Info

Paper received : 22.09.2016

Revised received: 12.11 .2016

Re-revised received : 22.12 .2016

Accepted : 24.12.2016
Abstract

Aim: The contribution of dehydrin is well known in drought stress tolerance in plants. The present study focus on identifying the markers linked to dehydrin protein for enhancing drought tolerance in chilli, Capsicum annuum.

Methodology: The publically available 1,15,000 plus EST collection of $C$. annuum were explored for identifying the presence of markers. A computer program was developed to read and analyse large fragments of nucleotide sequences and for locating the microsatellite repeat motifs in the EST collections of C. annuum. The program searched for exact motifs, a string of repeated units, with all possible combinations of motif occurrences.

Results: Based on conserved protein domains, several ESTs were identified with potential markers. Among them, one-third were predominated by dehydrin marker also having high sequence similarity. Three dehydrin markers were found to be highly conserved and robust, and were associated at high frequency with the DHN protein.

Interpretation: These potential dehydrin markers are linked directly to genes that encode trait specific characteristics and are highly useful for molecular breeding program for the selection of parental lines and for the development of crops tolerant to drought stress in changing climate scenario.
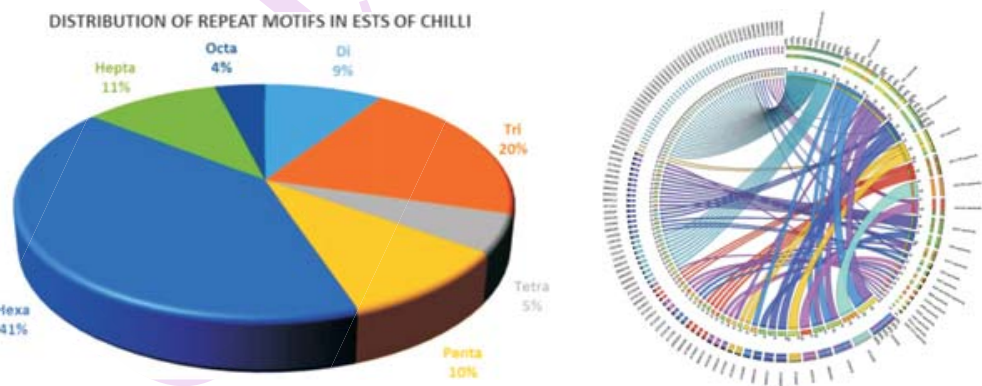

The identified motif in $C$. annuum using computer CIRCOS image showing dehydrin protein superfamily program. More of hexa motifs were found. predominated with one-third of total $\mathrm{C}$. annuum ESTs having markers.

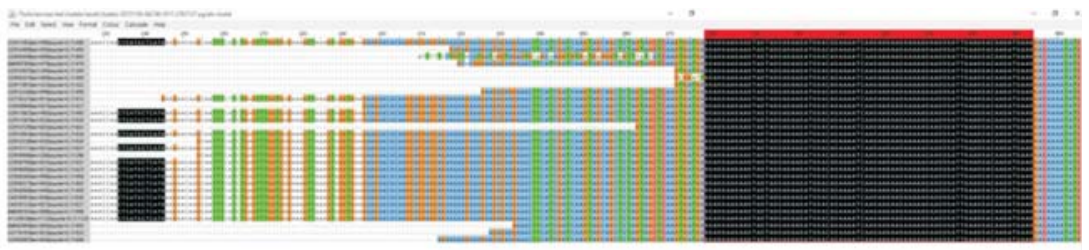

MSA showing highly conserved nature of the identified dehydrin marker in $C$. annuum 


\section{Introduction}

Chilli (Capsicum annuum) is a widely cultivated vegetable for its colour, flavor, spice and nutritional value. In India, chillies are grown in almost all the states and has great export potential. India has emerged as a leading producer and exporter of chilli, contributing one fourth of the world's production. It is rich in vitamin $A$, vitamin $C$, minerals and proteins. Any stress during flowering and fruiting stage adversely affects the productivity of the crop. Plants are stressed when exposed to high, as well as low temperature. When temperature falls below $15^{\circ} \mathrm{C}$ or exceeds $32^{\circ} \mathrm{C}$ for extended periods, growth and yield of the crop is affected. Chilli plants exposed to cold stress exhibit various symptoms, including flaccid stems and leaves, wilting and necrosis (Korkmaz et al., 2010, Airaki et al., 2012). Plants growing under stressed conditions respond by activating stress response genes. Stress conditions like drought, cold and high salinity cause dehydration in plants. Plants can withstand dehydration and protect themselves from the damaging effect due to a miraculous protein called Dehydrins (DHNs). DHN is a multi-family of proteins present in plants produced in response to drought and cold stress (Puhakainen et al., 2004). It also serves as cryoprotective agents and prevents damage of plant proteins under drought and cold temperature (Hughes and Graether, 2011). It is a great matter of interest to the scientists to research on DHNs, as it helps the plant to survive under water deprived condition. Under water stress condition, plant cells lose their water and reduce their turgor pressure (Holmberg and Bulow, 1998). However, stomatal closure helps in maintaining higher leaf water potential and hence, high leaf water content (Assaha et al., 2016). Chilli is one of the most sensitive crops to water deficit. Expression of dehydrin genes are regulated by abscisic acid,which tends to increase and play a role in dehydration tolerance (Allagulova et al., 2003). These protective measures are regulated by the changes in the expression level of genes. As genes and markers are physically close to one another on the same chromosome are tightly linked and if the markers linked with drought tolerant genes are located, it can be used in plant breeding program for imparting drought tolerance. Expressed Sequence Tags (ESTs) analysis is an efficient approach for identifying markers as EST derived markers are from the coding regions of the genome and are genetically associated with a trait of interest. The objective of the present study was focused to explore EST sequences of chilli, Capsicum annuum to identify dehydrin marker protein using computational methods.

\section{Materials and Methods}

EST sequences of Capsicum annuum : The EST sequence of Capsicum annuum was explored for markers associated with drought tolerance using computational methods. A computer program was exclusively developed to identify repeat motifs in EST sequences, based on mathematical algorithm that generates all possible combinations of motif occurrences viz., di, tri, tetra, etc. repeats in the EST sequence collections with the exemption of mono nucleotide repeats. The EST sequences, having repeat motif, were located by the program and it was found that the hexa motif was dominant among them. The graphical representation of identified ESTs in chilli having perfect repeat motifs (yellow color), specifically hexa toocta motifis is shown in Fig.1.

Conserved protein domain identification by comparative analysis: The identified ESTs were combined and generated as FASTA format file for batch processing. The computer program had located repeat regions in EST sequences of $C$. annuum along with their loci, which were extracted and generated as text file in FASTA format. Subsequently, it was processed as batch file for searching against Conserved Domain Database (CDD) for the presence of conserved protein domains associated with DHN proteins across several species. The sequences matching with the CDD were classified based on their respective protein superfamily.

\section{Results and Discussion}

The CDD search result revealed that approximately $6.25 \%$ of the identified EST sequences showed significant similarity to known stress tolerant proteins. The redundant ESTs were filtered and 128 unique ESTs had potential markers in $C$. annuum. These 128 non-redundant EST sequences with markers were categorized according to their traits having similarity with published proteins. It was found that these ESTs were spanned across 27 major proteins superfamilies associated with stress tolerance. Surprisingly, among them, the dehydrin protein superfamily was associated with 43 unique EST sequences which was pre-dominant. The EST and its associated protein superfamily relation is plotted and depicted (Fig. 2) using CIRCOS diagram (Krzywinski et al., 2009). ESTs are shown anticlockwise from top and protein super families are shown clockwise from top according to their volumes. From the above CIRCOS ideogram, the relationship between ESTs and protein superfamily and vice versa are indicated by different spectral bands. It was observed that one-third of the identified ESTs were predominated by dehydrin super family with 43 unique ESTs shown at top as blue spectral bands.

The identified dehydrin marker predominated among the analysed ESTs in C.annuum, which play a fundamental role in dehydration response of plants and adaptation to abiotic stresses. In wheat, a dehydrin gene (DHN-5) is inducible by either treatment with abscisic acid (ABA) or by a biotic stress such as drought and salinity (Ben et al., 2013).The EST sequences shown in Fig. 3 was associated with dehrydrative stress response that belongs to dehydrin superfamily. Multiple Sequence Alignment (MSA) was used in detecting the highly conserved motifs in identified EST sequences (Bilu et al., 2006). The sequences were aligned using Clustal W2, which revealed 


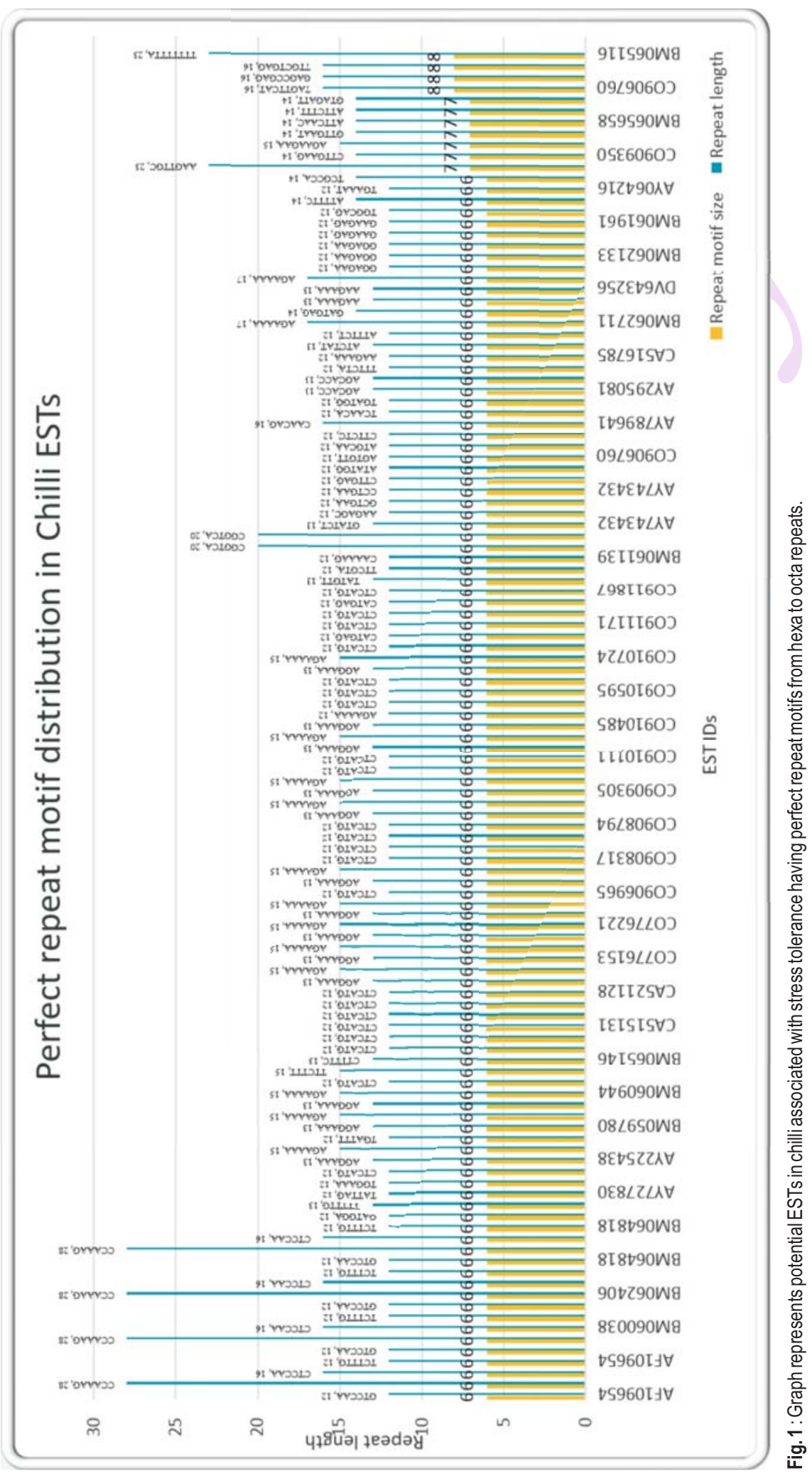




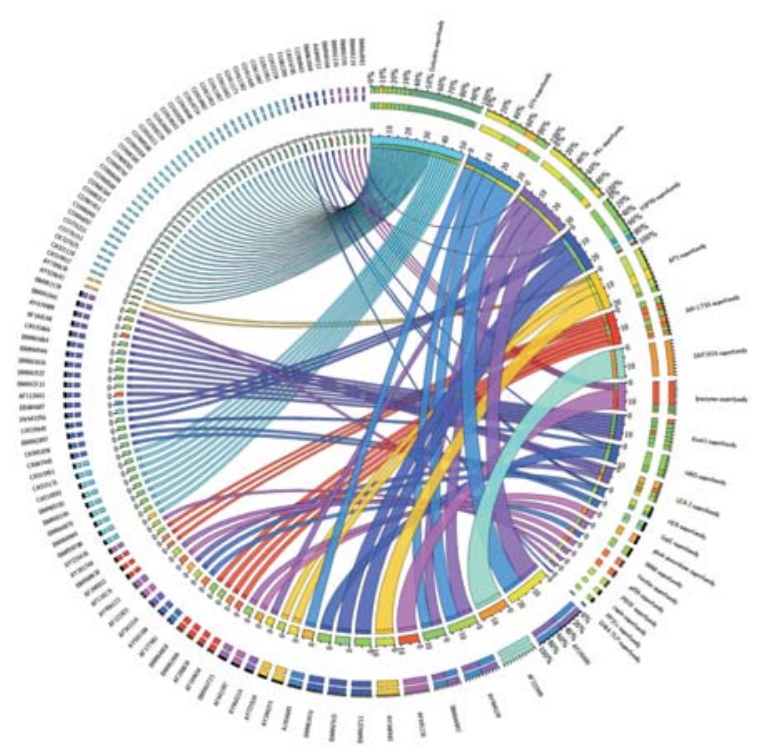

Fig. 2 : Ideogram representing C. annuum ESTs having potential markers and its respective superfamilies implicated in stress tolerance conserved marker region along with a hexa motif (CTCATG) repeated twice (12 bp pattern) preceding the marker sequences.

MSA of marker1 : The MSA (Fig.3) revealed the presence of 76 bp marker aligned without any gaps in 29 EST sequences which determines its robustness. The EST number and sequence length are shown in italics on the left side of the image in grey shade (Fig.3) and dehydrin marker alignment shown as black color and highlighted in red color as well.

MSA of marker 2 : From the MSA (Fig. 4), the 25 EST sequences aligned was having a $52 \mathrm{bp}$ of conserved marker region with tri repeat motif (AGG) found thrice in the marker sequence. Further, the same hexa motif (CTCATG) which was present in marker 1, is also repeated twice ( $12 \mathrm{bp}$ pattern) preceding the marker 2 as well (Fig. 4). This hexa motif (CTCATG) was repeated twice in two dehydrinmarker sequences and it was also highly conserved and present in the same locus with respect to marker sequence. This would be a potential signature for locating the dehydrin markers.

MSA of marker 3 : Six EST sequences, shown in Fig. 5, had 66

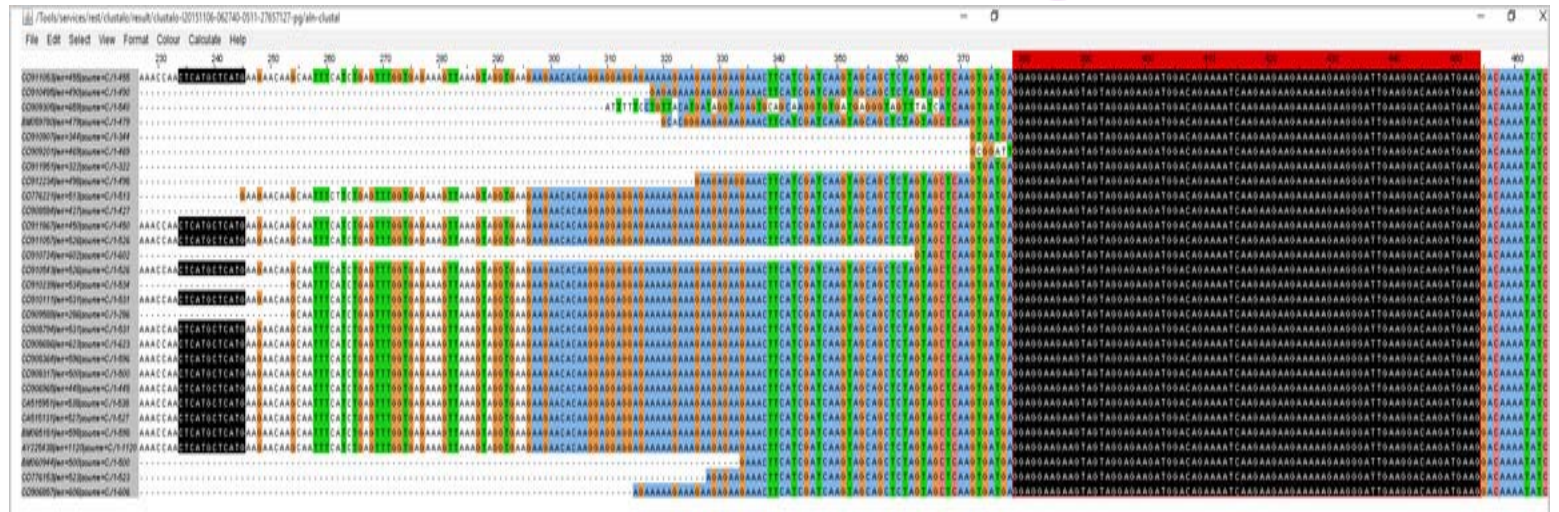

Fig. 3 : Dehydrin marker 1 shown in black color was present in 29 EST sequences having $76 \mathrm{bp}$ conserved region which is also highlighted in red color

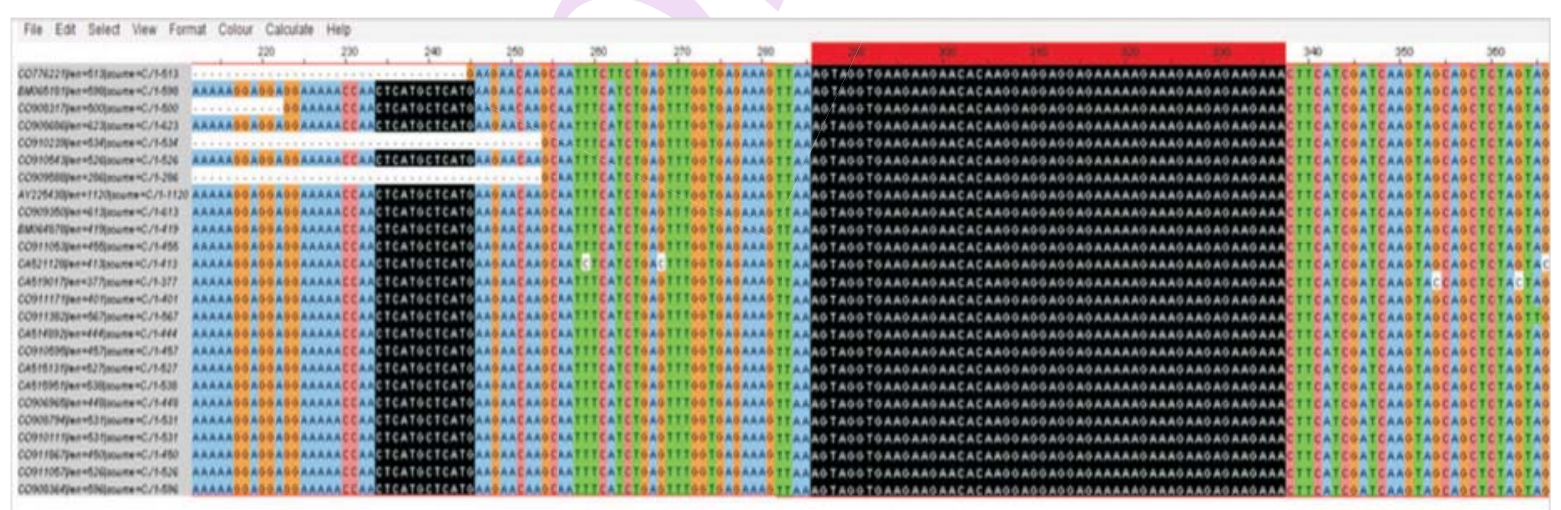

Fig. 4 : Dehydrin marker 2 (shown in black color) was present in 25EST sequences having 52bp conserved region (highlighted in red color) 


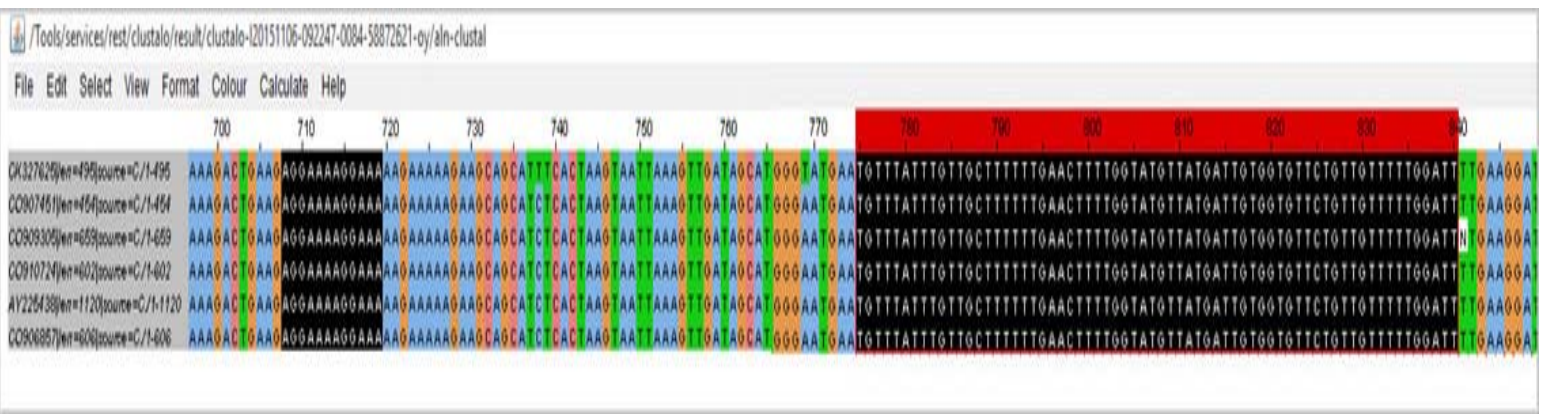

Fig. 5 : Dehydrin marker 3 (shown in black color) was present in 6 EST sequences having 66bp conserved region (highlighted in red color)

bp of conserved marker region. A hexa motif (AGGAAA) was also repeated twice, a $12 \mathrm{bp}$ pattern preceding the marker sequences. The MSA showed the conserved alignment of dehydrin markers associated with $\mathrm{DHN}$ protein.

Gene expression and protein accumulation of dehydrins have been observed in plants exposed to environmental stresses such as drought, cold and salinity, which have a dehydration component (Close, 1997). Among the C. annuum EST collections, three dehydrin markers associated with $\mathrm{DHN}$ protein were found to be highly robust and conserved in expressed region of the sequences associated with DHN. The association of DHN with stress tolerance suggest that dehydrins might be useful in improving the adaptation to drought (Lopez et al., 2003). The EST data analysis in Rangpur lime showed that among several proteins, dehydrinis associated with drought to increase their resistance to water stress (Raquel et al., 2007). DHN expression in oleaster plants was induced by drought stress and interacted with distinct cellular components involved in cell protection under water deficit likely through an ABA-dependent pathway (Chiappetta et al., 2015).

In plant species DHN genes exist in multiple copies, also high density of DHN genes are present in certain chromosomes (Liu et al., 2012). Concerns over the issue of identifying multiple copies of genes in whole genome using conventional approaches is reduced by computational methods and in silico analysis can significantly reduce the volume of laboratory work. In the present study, DHN markers were identified in multiple copies in C. annuum and some markers were found in large volume in ESTs that were part of the same cluster. Among the identified markers in complete EST collection of $C$. annuum, one-third were predominated by dehydrin which were having high frequency with the DHN protein. The identified potential dehydrin markers are highly useful for molecular breeding program for selection of parental lines and for the development of crops tolerant to drought stress in changing climate scenario.

\section{Acknowledgments}

The authors wish to thank Centre for Agricultural
Bioinformatics, the PI of Network Project on Agricultural Bioinformatics and Computational Biology for funding this research work. The authors also thank ICAR - Indian Institute of Horticultural Research and IASRI for technical support.

\section{References}

Allagulova, C.R., F.R. Gimalov, F.M. Shakirova and V.A. Vakhitov: The plant dehydrins: Structure and putative functions. Biochemistry (Moscow), 68, 945-951 (2003).

Airaki, M., M. Leterrier, R.M. Mateos, R. Valderrama, M. Chaki, J.B. Barroso, L.A. Del Río, J.M. Palma and F.J. Corpas: Metabolism of reactive oxygen species and reactive nitrogen species in pepper (Capsicum annuum L.) plants under low temperature stress. Plant Cell Environ., 35, 281-295(2012).

Assaha, D.V., L. Liu, A. Ueda, T. Nagaoka and H. Saneoka: Effects of drought stress on growth, solute accumulation and membrane stability of leafy vegetable, huckleberry (Solanum scabrum Mill.). J. Environ. Biol., 37, 107-14 (2016).

Ben, Amar S., H. Safi, M. Ayadi, J. Azaza, H. Khoudi, K. Masmoudi and F. Brini: Analysis of the promoter activity of a wheat dehydrin gene (DHN-5) under various stress conditions. Australian J. Crop Sci., 7, 1875-1883 (2013).

Bilu, Y., P.K. Agarwal and R. Kolodny: Faster algorithms for optimal multiple sequence alignment based on pairwise comparisons. IEEE/ACM Transact. Computat. Biol. Bioinform., 3, 408-422 (2006).

Chiappetta, A., A. Muto, L. Bruno, M. Woloszynska, M.V. Lijsebettens and M.B. Bitonti: A dehydrin gene isolated from feral olive enhances drought tolerance in Arabidopsis transgenic plants. Front. Plant Sci., 6, 392 (2015).

Close, T.J.: Dehydrins: A commonalty in the response of plants to dehydration and low temperature. Physiologia Plantarum., 100, 291-296(1997).

Holmberg, N. and L. Bülow: Improving stress tolerance in plants by gene transfer. Trends Plant Sci, 3, 61-66 (1998).

Hughes, S. and S.P. Graether: Cryoprotective mechanism of a small intrinsically disordered protein. Protein Sci., 20, $42-50$ (2011).

Korkmaz, A., Y. Korkmaz and A.R. Demirkıran: Enhancing chilling stress tolerance of pepper seedlings by exogenous application of 5aminolevulinic acid. Environ. Exp. Bot., 67, 495-501(2010).

Krzywinski, M., J. Schein, I. Birol, J. Connors, R. Gascoyne, D. Horsman, S.J. Jones and M.A. Marra: Circos: An information aesthetic for comparative genomics. Genome Res., 19, 1639-1645 (2009). 
Liu, C.C., C.M. Li, B.G. Liu, S.J. Ge, X.M. Dong, W. Li, H.Y. Zhu, B.C. Wang and C.P. Yang: Genome-wide identification and characterization of a dehydringene family in Poplar (Populustrichocarpa). Plant Mol. Biol. Rep., 30, 848-859(2012).

Lopez, C., G.M. Banowetz, C.J. Peterson and Kronstad: Dehydrin expression and drought tolerance in seven wheat cultivars. Crop Sci., 43, 577-582(2003).

Puhakainen, T., M.W. Hess, P. Mäkelä, J. Svensson, P. Heino and E.T.
Palva: Overexpression of multiple dehydrin genes enhances tolerance to freezing stress in Arabidopsis. Plant Molec. Biol., 54, 743-53(2004).

Raquel, L. Boscariol-Camargo, I.J. Berger, A.A. Souza, A.M. do Amaral, E.F. Carlos, J.F. Astúa, M.A. Takita, M.L.P.N. Targon, C.L. Medina, M.S. Reis and M.A. Machado: In silico analysis of ESTs from roots of Rangpur lime (Citrus limonia Osbeck) under water stress. Genet. Molec. Biol., 30, 906-916 (2007). 